\section{Mehr Sicherheit durch die Therapieallergene- Verordnung}

- Seit dem November 2008 gilt in Deutschland die Therapieallergene-Verordnung. Diese regelt, dass die häufigsten Allergene zur spezifischen Immuntherapie in Zukunft einer Zulassung bedürfen. Welche praktischen Auswirkungen dies hat, erklärt Prof. Dr. Randolf Brehler.

? Was ist das Anliegen der Therapieallergene-Verordnung?

Brehler: Die Verordnung regelt, dass häufig verwendete Allergenextrakte, wie Süßgräser, Baumpollen, Hausstaubmilben, Bienen- und Wespengift, dem normalen Zulassungsprozedere unterzogen werden müssen. Ziel ist der Nachweis der Sicherheit und Effektivität unter Studienbedingungen. Als Allergologe begrüße ich die Neuregelung, da wir sicher sein können, dass künftig nur Extrakte auf den Markt kommen, deren Wirksamkeit belegt werden konnte.

? Was hat sich in der bisherigen Praxis geändert?

Brehler: Die Herstellerfirmen mussten bis zum 1. Dezember 2010 für Präparate, die zugelassen werden sollen, die entspre- chenden Anträge bei den Behörden vorlegen. Zur Behebung von Mängeln gibt es dann ein weiteres Jahr Zeit. Im Anschluss müssen entsprechende Studien durchgeführt werden. Bereits zugelassene Allergene können weiter so verschrieben werden wie bisher.

? Einige Firmen bieten einen großen Teil an bereits zugelassenen Fertigarzneimitteln an. Welchen Vorteil hat dies für die tägliche Praxis?

Brehler: Da die Zulassungen bereits vorliegen, kann von einer langfristigen Verfügbarkeit dieser Produkte ausgegangen werden. Ihre Effektivität und Sicherheit ist entsprechend der früher gültigen Bedingungen geprüft worden. Ebenso ist der vorliegende Zulassungsstatus erfahrungsgemäß bei der Diskussion der Erstattungsfähigkeit eine gute Argumentationshilfe.

? Hyposensibilisierungen sind langfristige Behandlungen. Kann der Arzt jetzt verlässlich planen, dass er die Therapie mit einem noch nicht zugelassenen Präparat bis zu Ende durchführen kann?

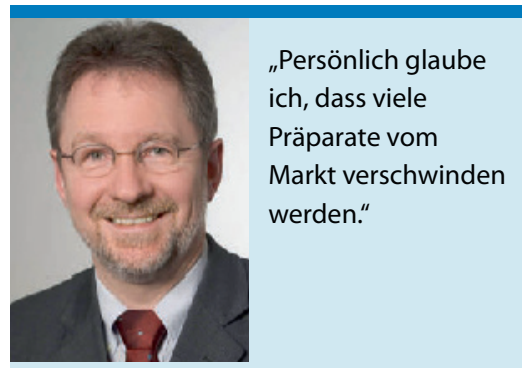

Prof. Dr. Randolf Brehler

Klinik und Poliklinik für Hautkrankheiten, Universitätsklinikum Münster

Brehler: Diese Frage ist momentan nur schwer zu beantworten. Es ist beispielsweise vorstellbar, dass ein Hersteller zwar eine Zulassung anstrebt, diese dann aber aus Kostengründen nicht durchführen kann. Auch kann nicht vorausgesagt werden, ob für alle Präparate der Wirksamkeitsnachweis in den geforderten Studien gelingen wird. Persönlich glaube ich, dass viele Präparate vom Markt verschwinden werden.

! Herr Professor Brehler, vielen Dank für das Gespräch.

Das Interview führte Bettina Reich

Nach Informationen von Allergopharma

\title{
Mit Hyaluronsäure-Tabletten unangenehme Halsbeschwerden effektiv lindern
}

— Hals- und Rachenentzündungen zählen mit rund sechs Millionen neu diagnostizierter Fälle pro Jahr zu den häufigsten Krankheiten überhaupt.

Jetzt liegen die Ergebnisse einer Beobachtungstudie zur Wirksamkeit von Halstabletten mit Hyaluronsäure vor (GeloRevoice ${ }^{\circledR}$ ). Die Tabletten bilden einen Hydrogel-Komplex aus und bedecken so wie ein zweiter Schutzfilm die betroffene Schleimhaut. Die Studie ergab, dass sich die Hauptsymptome nach rund fünftägiger Behandlung um 76\% besserten. Dies betonte Prof.. Hans Behrbohm, Berlin, Leiter dieser prospektiven monozentrischen Anwendungsbeobachtung. Die teilnehmenden 20 Patienten litten unter den Hauptsymptomen Heiserkeit, Kratzen im Hals, Hustenreiz, trockene Mund- und Rachenschleimhaut und Halsschmerzen. Zielkriterien waren das Ausmaß der Bedeckung sowie die Verbleibdauer des Hydrogel-Komplexes im Mund- und Rachenraum. Zur Darstellung des Symptomverlaufs dienten Patientenbefragungen am Beginn und Ende der rund fünftägigen Behandlung. Bereits zehn Minuten nach Tabletten-Einnahme verteilt sich nach videoendoskopischen Befunden der entstandene Hydrogel-Komplex relativ gleichmäßig in Mundhöhle und Oropharynx, so Behrbohm. Die empfundene Verbleibdauer des Hydrogels von 30 Minuten bis über eine Stunde nach Einnahme der Tablette bezeichnete Behrbohm als günstig. Insgesamt gingen die Beschwerden wäh- rend der Behandlung laut Patientenbefragung um $76 \%$ zurück. Den deutlichsten Effekt registrierten die behandelnden Ärzte beim Symptom „Halsschmerzen", die sich um $85,3 \%$ reduzierten. Etwa ein Viertel der Patienten war nach Behandlungsende komplett beschwerdefrei, betonte Behrbohm. Dieser Beobachtungsstudie mit geringer Teilnehmerzahl soll eine große Anwendungsstudie mit rund 1.200 Probanden folgen, hieß es von Seiten der Veranstalter.

Wolfgang Zimmermann

Pressekonferenz ${ }_{\text {"GeloRevoice }}{ }^{\circledR}$ im Spiegel der klinischen Forschung ", im Rahmen der EXPOPHARM 2010, München, 8. Oktober 2010; Veranstalter: Pohl-Boskamp 